\title{
Degeneration of solventogenic Clostridium strains monitored by Fourier transform infrared spectroscopy of bacterial cells
}

\author{
KC Schuster ${ }^{1}$, R Goodacre ${ }^{2}$, JR Gapes ${ }^{1}$ and M Young ${ }^{2}$ \\ ${ }^{1}$ Institute of Chemical Engineering, Fuel and Environmental Technology, Vienna University of Technology, Vienna A1060, \\ Austria; ${ }^{2}$ Institute of Biological Sciences, Cledwyn Building, University of Wales Aberystwyth, Aberystwyth, Ceredigion SY23 \\ $3 D D, U K$
}

\begin{abstract}
Strain degeneration in solventogenic clostridia is a known problem in the technical acetone-butanol fermentation bioprocess, especially in the continuous process mode. Clostridial strain degeneration was studied by Fourier transform infrared (FT-IR) spectroscopy of the bacterial cells. Degenerative variant formation in two strains, Clostridium beijerinckii NCIMB 8052 and Clostridium species AA332, was detected spectroscopically. Colonies on solid media were sampled, or assayed directly in situ by IR microscopy. It has previously been shown that the distinctive acidogenic and solventogenic physiological phases of Clostridium acetobutylicum in liquid medium can be discriminated by FT-IR spectroscopy. This was confirmed here for C. beijerinckii NCIMB 8052 . The proportion of degenerate cells in a mixed population in liquid medium could be quantified, as the spectral features change in different ways during the normal growth cycle of wild type organisms and degenerate variants in batch culture. This opens a new perspective for physiology-based process monitoring and control, especially of the continuous acetone-butanol fermentation. Journal of Industrial Microbiology \& Biotechnology (2001) 27, 314-321.
\end{abstract}

Keywords: Acetone-butanol fermentation; Clostridium; solventogenic fermentation; strain degeneration; FT-IR spectroscopy; fermentation process monitoring

\section{Introduction}

Solvent production in Clostridium species depends on the complete function and finely balanced regulation of a complex metabolic network, involving more than a dozen enzymatic functions [8,31]. In the technological application of solvent production, i.e., the acetone-butanol fermentation, the optimal operation of this metabolic network is crucial for yield and productivity, and thus for economic success [12,33]. During the course of a solventproducing batch fermentation, the initial phase of exponential growth is associated with the production of volatile fatty acids (acetate and butyrate). When the concentration of these acids, or the culture $\mathrm{pH}$, has reached a certain threshold, clostridial metabolism switches to solvent production [19]. At this stage, many of the organisms present stop growing and endospore formation is initiated.

The term "degenerate" has traditionally been employed in connection with the acetone-butanol fermentation to describe both strains and cultures that produce no solvents. This is potentially a source of considerable confusion. We reserve the term degenerate to describe the inherent and heritable properties of organisms or strains. It therefore follows that degeneration results from genetic change (genetic instability or mutation). It is frequently connected with a loss of the ability to sporulate [7]. In Clostridium acetobutylicum, genes encoding essential enzymes in the solventogenic pathways are located on a megaplasmid whose loss results in a degenerate phenotype $[6,7,37]$. In Clostridium saccharoperbutylacetonicum, degenerate mutants were identified with a defect in

Correspondence: Prof M Young, Institute of Biological Sciences, Cledwyn Building, University of Wales Aberystwyth, Aberystwyth, Ceredigion SY23 3DD, UK Received 6 October 2000; accepted 20 April 2001
NADH formation from pyruvate [16]. Clostridium beijerinckii forms degenerate variants even more frequently than $C$. acetobutylicum, but the underlying mechanism remains unclear. An insertion sequence has recently been characterised in this organism but there is no evidence that it plays a prominent role in degeneration [23].

In contradistinction to the process of degeneration, cultures of solventogenic organisms sometimes fail to produce solvents for physiological or regulatory reasons [24]. This behaviour has been termed "acid crash," an expression used in the former solvent industry (David Jones, personal communication, 1997). Conditions favouring or preventing the acid crash have been variously described $[4,21,24]$. Acid crash often occurs when bacteria are growing at or close to their maximum growth rate or in bacteria with a very high metabolic rate $[9,21]$. Under these conditions, the switch from acid to solvent production fails to occur before the acids accumulate to toxic levels [10,20,24].

The process of degeneration occurs during repeated vegetative subculture $[21,22]$, on solid media $[1,22,40]$ and most especially during continuous cultivation $[2,3,5,11,30,40]$. Technological development of the acetone-butanol fermentation is tending toward the establishment of continuous processes for economic reasons [12]. During long-term continuous solvent fermentations [13], degeneration is an intrinsic problem. In a working environment, it is difficult to detect degeneration and discriminate it from a poor performance, or acid crash, due to unfavourable fermentation conditions. This was the motivation for the present study. There is good evidence from previous studies that the slow and progressive loss of solvent productivity is due to the formation of a degenerate subpopulation in the culture. Distinct variants can be isolated from continuous cultures, and discriminated on solid medium [40]. The degenerate population gradually takes over, as these bacteria keep 
growing, whereas the solvent-producing organisms in the wildtype population slow down or stop growth and initiate sporulation [21].

In this investigation infrared spectroscopy was used to analyse bacterial populations. Naumann and coworkers [28] first employed Fourier transform infrared (FT-IR) spectroscopy of dried films of cells in transmission mode on translucent carriers to identify organisms. It was used recently to analyse the physiological status (acidogenic vs. solventogenic phase) of C. acetobutylicum cultures [34]. (The strain used there was reported to be $C$. beijerinckii NRRL B592, but it turned out later that one experiment has been actually conducted with C. acetobutylicum. However, the physiological behaviour and the spectral changes of the two organisms were very similar.) The physiological state associated with acid crash could also be differentiated (Grube, Schuster, and Gapes, manuscript submitted). An alternative method for analysis of bacterial cells was developed by Goodacre et al. [14] who employed the diffuse reflectance-absorbance mode. This protocol was used in the present study.

\section{Materials and methods}

\section{Strains and strain maintenance}

C. beijerinckii NCIMB 8052 and C. acetobutylicum AA332 stocks were stored at $-80^{\circ} \mathrm{C}$ with $20 \%$ glycerol as cryoprotectant. AA332, a solventogenic organism of unknown origin was kindly provided by P. Claassen and A. Lopez-Contreras (ATO, Wageningen, the Netherlands). Degenerate variants of $C$. beijerinckii NCIMB 8052 and C. acetobutylicum AA332 were isolated during this study from colony outgrowths of the respective normal (wild type) strains. They were kept on solid medium by passage every few days.

\section{Culture media and culture conditions}

Agar-solidified clostridial basal medium (CBM) [29] was used for short-term culture maintenance and colony counts. Cultures $(25 \mathrm{ml})$ in liquid medium were grown in tubes containing modified semisynthetic medium (MSS), containing $60 \mathrm{~g} / 1$ glucose, $5 \mathrm{~g} / 1$ yeast extract, and salts according to Ref. [35]. The first liquid culture passage of each series of experiments was inoculated from a single colony. All cultures were grown in an anaerobic cabinet in an atmosphere of $10 \%$ hydrogen, $10 \% \mathrm{CO}_{2}$ and $80 \%$ nitrogen at $37^{\circ} \mathrm{C}$ ( solid media) or $34^{\circ} \mathrm{C}$ (liquid media).

Colonies of normal and degenerate organisms were differentiated by their morphology according to Ref. [40]. Culture samples were serially diluted using a holding buffer $(25 \mathrm{mM} \mathrm{K}$ phosphate, $\mathrm{pH} 7,1 \mathrm{mM} \mathrm{MgSO} 4$ ). Liquid cultures were performed in two or three replicates each.

\section{Analysis of fermentation medium components}

After removal of bacteria by centrifugation, solvents and acids were determined by gas chromatography according to Ref. [13]. Cell density was estimated as optical density (OD) by light scattering in a spectrophotometer at $615 \mathrm{~nm}$.

\section{Preparation of cell samples and FT-IR analysis}

Samples of cells grown either on solid medium or in liquid medium were taken under anaerobic conditions. Cells from liquid culture samples were harvested by centrifugation. Cell pellets were washed twice in physiological saline $(0.9 \% \mathrm{NaCl})$, then diluted in saline. Colonies from solid media were suspended in physiological saline for washing, then treated like the samples from liquid media. Portions of the bacterial slurries $(5 \mu \mathrm{l})$ were evenly applied onto an aluminium plate. Prior to analysis triplicate samples were ovendried at $50^{\circ} \mathrm{C}$ for $30 \mathrm{~min}$. IR spectra were collected using a Bruker IFS28 FT-IR spectrometer (Bruker Spectrospin, Banner Lane, Coventry, UK) equipped with an MCT (mercury-cadmiumtelluride) detector cooled with liquid $\mathrm{N}_{2}$. The aluminium plate was loaded onto the motorised stage of an adapted reflectance TLC accessory [38]. The spectrometer was controlled (using OPUS version 2.1 software running under IBM O/S2 Warp provided by the manufacturers) to collect spectra over the wave number range 4000 to $600 \mathrm{~cm}^{-1} 20$ spectra per second were acquired. The spectral resolution used was $4 \mathrm{~cm}^{-1}$. To improve the signal-tonoise ratio, 256 spectra were averaged. Each sample was thus represented by a spectrum containing 882 data points and spectra were displayed in terms of absorbance as calculated from the reflectance-absorbance spectra using the Opus software (which is based on the Kubelka-Munk theory [15]).

For measurements directly from agar plates a Bruker infrared microscope (A590) was used and the IR beam was focused onto the surface of the clostridial colonies. Spectra were acquired with an Opticon $\times 15$ (4NA) objective such that the sampling area was $0.785 \mathrm{~mm}^{2}$ (diameter $0.5 \mathrm{~mm}$ ). Spectra were also collected over the wave number range 4000 to $600 \mathrm{~cm}^{-1}$. The spectral resolution used was $16 \mathrm{~cm}^{-1}$ and 128 spectra were averaged.

To minimise problems arising from baseline shifts, the first derivatives of the original FT-IR spectra were smoothed using the Savitzky-Golay algorithm [32] using five-point smoothing.

\section{Spectrum evaluation by derivatives}

For a better visual resolution of spectral features, original FT-IR spectra were derivatized, then vector normalized, and derivatized again, resulting in second-derivative spectra that are normalised to the total signal intensity. These second-derivative spectra were plotted on an inverted abscissa scale, so that the positive peaks appearing correspond to positive peaks in the original spectra.

\section{Cluster analysis}

The initial stage involved reduction of the multidimensional FT-IR data by principal components analysis [17]. Principal component analysis is a well-known technique for reducing the dimensionality of multivariate data, while preserving most of the variance. The first principal component describes the largest amount of the variance, the second describes somewhat less, and so on. The set of the first few principal components can usually give a quite good approximation to the total variance. Principal components analysis was performed according to the NIPALS algorithm [39]. Discriminant function analysis (also known as canonical variates analysis) then discriminated between groups on the basis of the retained principal components and the a priori knowledge of those spectra that were replicates, and thus this process does not bias the analysis [25].

\section{Partial least squares}

When the desired targets associated with each of the spectra are known, then a supervised data evaluation algorithm may be used. The goal of supervised learning is to find a mathematical model that 
will correctly associate the inputs with the targets. This is usually achieved by minimizing the error between the target and the model's response. Briefly, a training set of spectra with known ratios of normal to degenerate cells was used to form the model. A validation set (also of spectra from samples with known normal to degenerate cell ratios) was used in conjunction with the training set to establish the optimum model. Finally, an independent test set, consisting of spectra that were not used by the model creation program at any time, was used to test the effectiveness of the calibrated system. The partial least squares algorithm described the targets as a linear combination of so-called factors (which play a similar role to the principal components in principal component analysis). The first few factors usually describe most of the correlation between the targets and the spectra.

In this case, the training set were samples from time 1 (from the growth phase), containing $0 \%, 10 \%, 20 \%, 30 \%, 40 \%, 50 \%, 60 \%$, $70 \%, 80 \%, 90 \%$ and $100 \%$ degenerate cells. The cross-validation set was also from time 1 and contained $5 \%, 15 \%, 25 \%, 35 \%, 45 \%$, $55 \%, 65 \%, 75 \%, 85 \%$ and $95 \%$ degenerate cells. The independent test set were 21 samples from time 2 (from the stationary phase), which contained $0-100 \%$, in $5 \%$ steps, degenerate cells.

All partial least squares analyses [26] were carried out using an in-house program [18], which runs under Microsoft Windows NT on an IBM-compatible PC. The first stage was preparation of the data. This was achieved by presenting the training set as two data matrices to the program: X, which contains the FT-IR spectra, and $\mathrm{Y}$, which represents the percentage of degenerate to normal cells. The $\mathrm{X}$-data were mean-centered and scaled in proportion to the reciprocal of their standard deviations [26].

The next stage was generation of the calibration model. The method of validation used was full cross - validation, via the leaveone-out method [26]. This technique sequentially omits one sample from the calibration; the partial least squares model is then redetermined on the basis of this reduced sample set. The ratio of the omitted sample is then predicted with the use of this model. This method is required to determine the optimal size of the calibration model, so as to obtain good estimates of the precision of the multivariate calibration method (i.e., neither to under- nor overfit predictions of unseen data) [36].

To choose the optimal number of latent variables (partial least squares factors) to use in predictions after the model was calibrated, cross - validation was employed. This was achieved by choosing the number of partial least squares factors used in the predictions that gave the minimum root mean square error for the cross - validation set.

\section{Results and discussion}

\section{Analysis of "harvested" normal and degenerate colonies}

Colonies of Clostridium sp. AA332 and C. beijerinckii NCIMB 8052 were grown on solid CBM medium and harvested after 45 and $110 \mathrm{~h}$. C. beijerinckii NCIMB 8052 was used as its degeneration behaviour has already been studied extensively [40]. Clostridium sp. AA332 was chosen because it is particularly prone to degeneration (M. Young, K. Jennert, unpublished results). At $45 \mathrm{~h}$, the colonies of both strains appeared more or less homogeneous among themselves by morphology, but after $110 \mathrm{~h}$, the colonies of Clostridium sp. AA332 were clearly of two morphologically different types. One type was small, rounded and

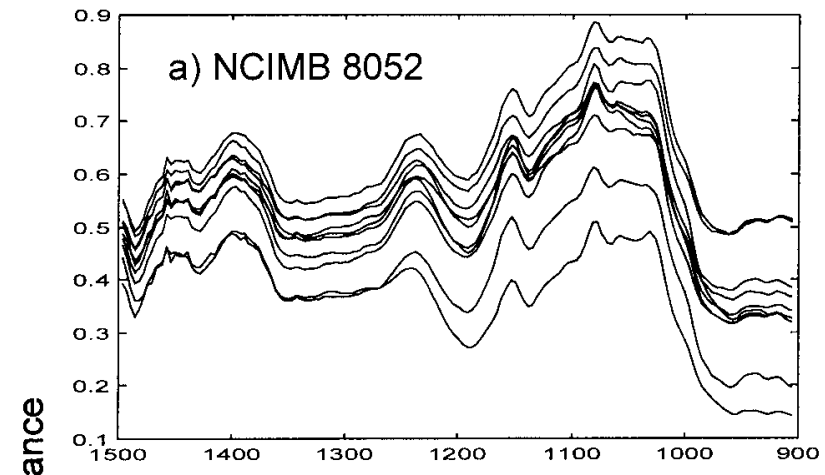

잉

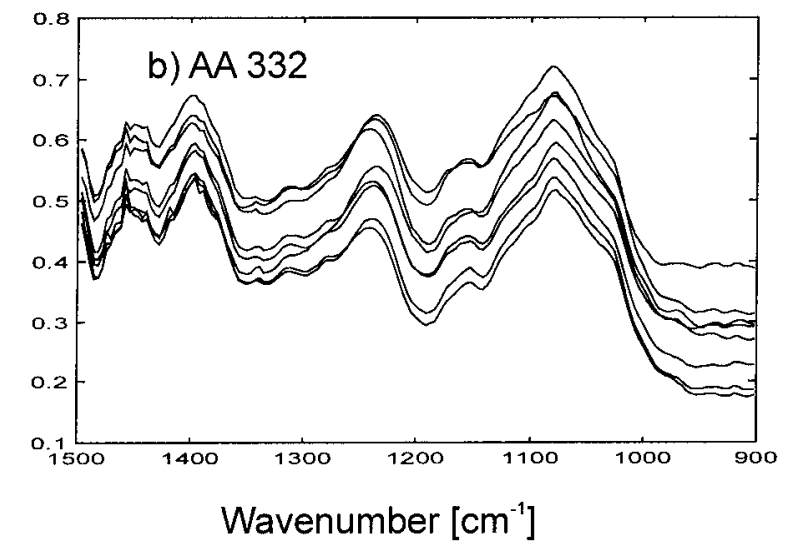

Figure 1 FT-IR spectra of single colonies of the two strains: (a) C. beijerinckii NCIMB 8052 and (b) Clostridium strain AA332 after cultivation for $45 \mathrm{~h}$ on solid medium, in the "fingerprint" spectral range of $1500-900 \mathrm{~cm}^{-1}$.

opaque, whereas the other was larger, translucent and had an irregular shape. These two distinctive colonial morphologies are characteristic of normal and degenerate bacteria in $C$. beijerinckii [40]. The colonies of $C$. beijerinckii NCIMB 8052, however, retained a uniform morphology.

IR spectra were taken from 7 to 10 different colonies of each strain. Some colony samples were prepared for IR measurements in three replicates. Eight representative spectra of single colonies are shown in Figure 1. Spectra from different colonies of the same strain were quite uniform, and quite different from those of colonies of the other strain, especially in the carbohydrate region (1200 to $900 \mathrm{~cm}^{-1}$ ).

At $45 \mathrm{~h}$, differences between the two strains were clearly detected by principal component analysis of the FT-IR spectra (Figure 2a), but no degenerate colonies could be detected either by morphology or in the spectra. However, after cultivation for $110 \mathrm{~h}$ on solid medium some colonies from strain AA332 showed completely degenerate morphology and others had a normal center with degenerate outgrowths. An analysis of the similarity of spectra from colonies of the two strains and the degenerate variant of AA332 are shown in Figure 2b. The colonies of NCIMB 8052 show a certain diversity at this stage of development, but no clear separation into different clusters. The difference between NCIMB 8052 and AA332 is quite clear cut. Moreover, the splitting of AA332 into two subpopulations, corresponding to normal and degenerate colony morphology, is clearly visible. Concerning the latter, colonies with completely degenerate morphology cluster together with the degenerate outgrowths of normal colonies. 
a) Colonies at $45 \mathrm{~h}$

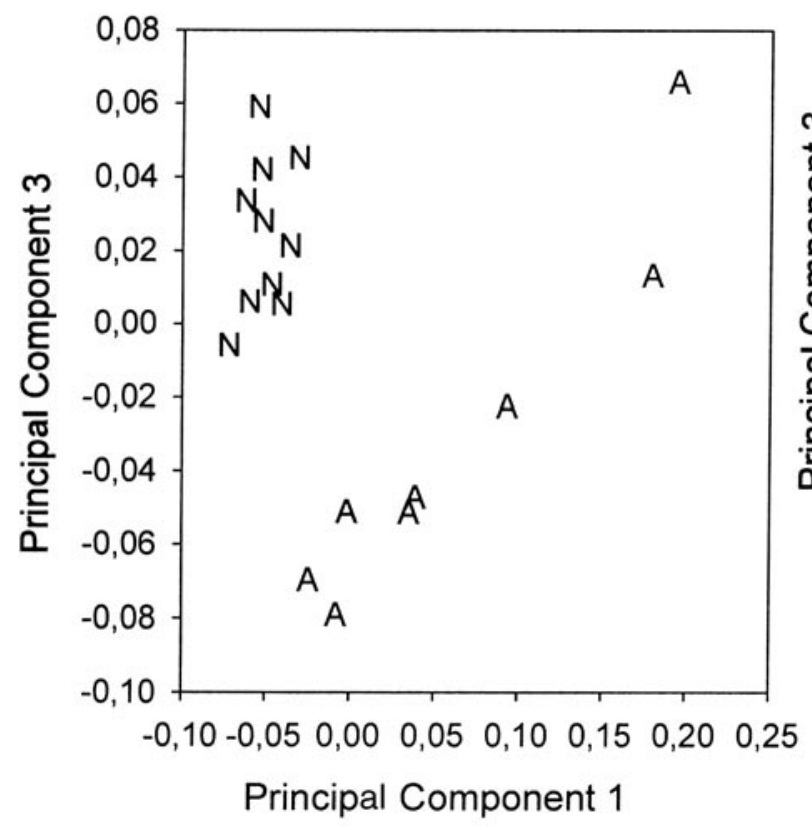

b) Colonies at $110 \mathrm{~h}$ 317

Figure 2 Principal components analysis of the spectra of colonies. Data analysis on first-derivative spectra. The whole spectral range recorded was taken into account. (a) Time 45 h: Clostridium strain AA332 (data points "A") and NCIMB 8052 (data points "N") were differentiated by the principal components 1 and 3. Principal components 1,2 and 3 accounted for $48.5 \%, 20.0 \%$ and $14.8 \%$ of the variance, respectively (total $=83.3 \%$ ). (b) Time 110 h: C. beijerinckii NCIMB 8052 (data points "N"), normal colonies of Clostridium strain AA332 (data points "A"), and strain DA, the degenerate variant of strain AA332 (data points "D": whole colonies with degenerate morphology; data points "G": colonies with degenerate outgrowths) were differentiated by the principal components 1, 2 and 3. Principal components 1,2 and 3 accounted for $55.7 \%, 20.2 \%$, and $12.5 \%$ of variance, respectively ( total $=88.4 \%$ ).

\section{Analysis of a degenerating colony on solid medium by infrared microscopy}

In order to analyse directly a degenerating colony of $C$. beijerinckii NCIMB 8052 on solid medium, an agar plate was placed under the IR microscope. The IR beam was focused onto the colony. Spectra were taken across the agar surface and a colony with a degenerate outgrowth, on a path shown in Figure 3. Principal component analysis of the spectra could clearly detect the difference between the normal part of the colony and the degenerate outgrowth (not shown).
From such a colony outgrowth, a degenerate variant of C. beijerinckii NCIMB 8052 was isolated for the following experiments. The strain was denoted strain $\mathrm{DN}$.

\section{Spectral changes during growth and solvent formation} of C. beijerinckii NCIMB 8052

In an earlier study [34], it was observed that FT-IR spectra of C. beijerinckii NRRL B592 and C. acetobutylicum change significantly during growth and solvent production in batch culture experiments. The strains [34] show a major difference to

\section{Degenerate outgrowth of colony}

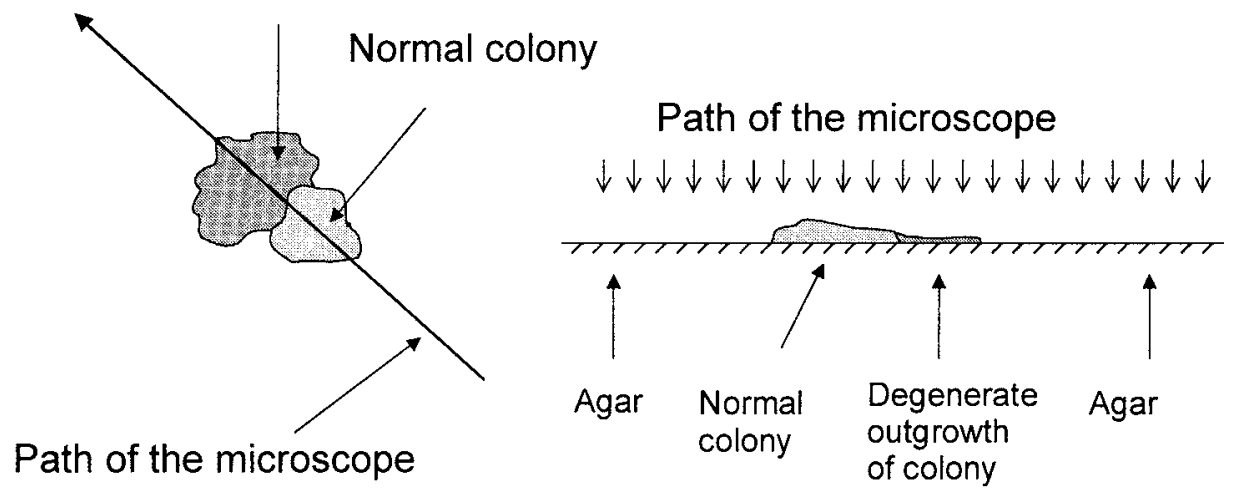

Figure 3 Analysis of a single colony using reflectance FT - IR by an IR microscope coupled to the FT - IR spectrometer. Schematic drawings: left, path of the microscope line scan over the agar surface and colony; right, cross section through the agar surface. 
318

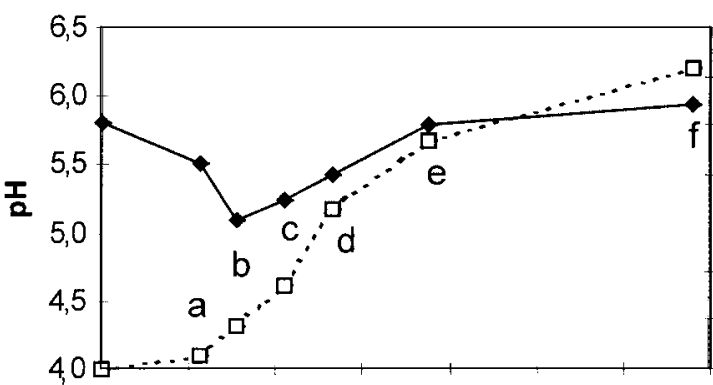

7,0

$6,0 \mathrm{5}$

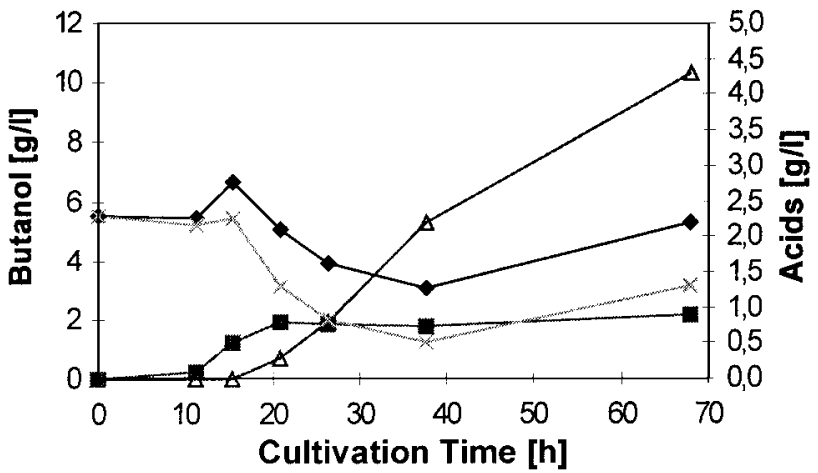

Figure 4 Growth and production curve of $C$. beijerinckii NCIMB 8052. Average values from two parallel experiments under identical conditions are shown. Letters "a" to "f" indicate the time points of sampling for the IR spectroscopic analysis (spectra in Figure 5 and data points in Figure 6). (a) $\diamond, \mathrm{pH}$ value and $\square$, growth by optical density. (b) Products: $\triangle, n$-butanol; $\diamond$, total acids; butyrate; $\times$, acetate.

C. beijerinckii NCIMB 8052 in their physiology: they stop growing at the shift to the solventogenic phase, whereas $C$. beijerinckii NCIMB 8052 continues to grow throughout the whole cultivation experiment (Figure 4). However, the change in OD is not necessarily proportional to the biomass dry matter concentration due to cell differentiation, which changes the optical properties of cells. The culture was inoculated 1/1000 with a liquid preculture (passage 1, $12 \mathrm{~h}$ old), which had been inoculated from a single colony on solid medium. The time course of product formation (Figure 4 ) showed the typical biphasic pattern. Starting with acid production, the culture later shifted to solvent production. In this latter phase, some of the acetate present in the medium was converted into solvents.

There were significant changes in FT-IR spectra during the growth curve of $C$. beijerinckii NCIMB 8052 in batch culture (Figure 5). Bands are assigned according to Ref. [27]. Changes in regions of $1407 \mathrm{~cm}^{-1}$ could be attributed to an increasing lipid content in late culture phases. This effect was previously observed with $C$. acetobutylicum [34]. In the carbohydrate region (1200 to $900 \mathrm{~cm}^{-1}$ ), changes were very significant. Some can be attributed to the formation of a starch-like storage carbohydrate (granulose) that was also detected in the light microscope with iodine-stained samples. The fairly constant size of the band at $1241 \mathrm{~cm}^{-1}$, corresponding to phosphate esters in nucleic acids, is noteworthy. This band appeared to be dependent on the growth phase in C. acetobutylicum; growth ended at the solvent shift and the nucleic acid band decreased drastically. Here, the band stayed rather constant, consonant with continuing growth throughout the solventogenic phase (Figure 4).

Discriminant function analysis of the spectra (Figure 6) showed pronounced differences over the bacterial growth curve. Samples from the acidogenic exponential growth phase and early solventogenic phase ( samples a, b, and c) formed a discrete cluster in the space of discriminant functions 1 and 2, whereas samples from the solventogenic phase (samples d, e, and f) gave separate clusters different from each other.

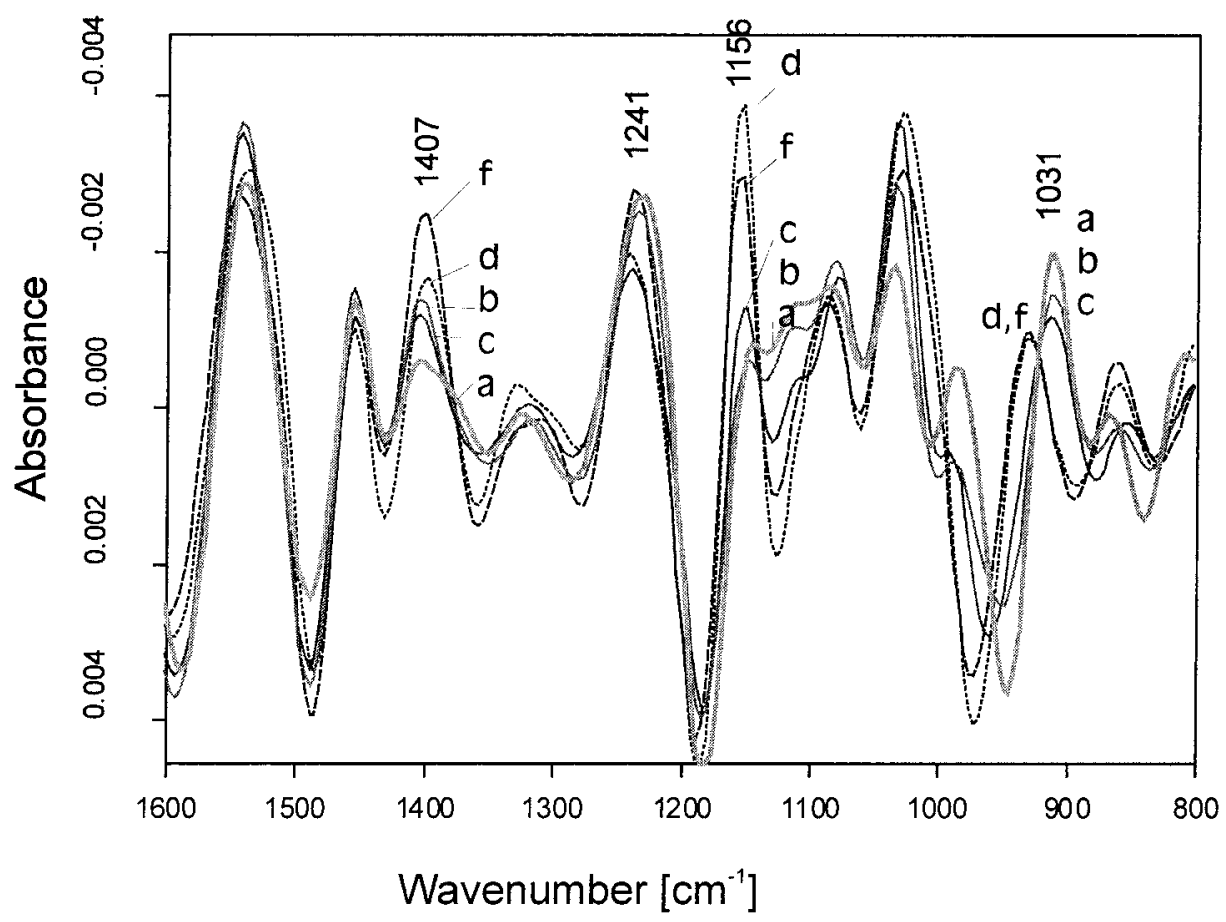

Figure 5 Second derivative FT - IR spectra over the growth curve of $C$. beijerinckii NCIMB 8052. Sampling time points are marked with letters "a" to " $\mathrm{f}$ " corresponding to those indicated in Figure 4. Spectra from one of the two parallel experiments (experiment A) are shown. 


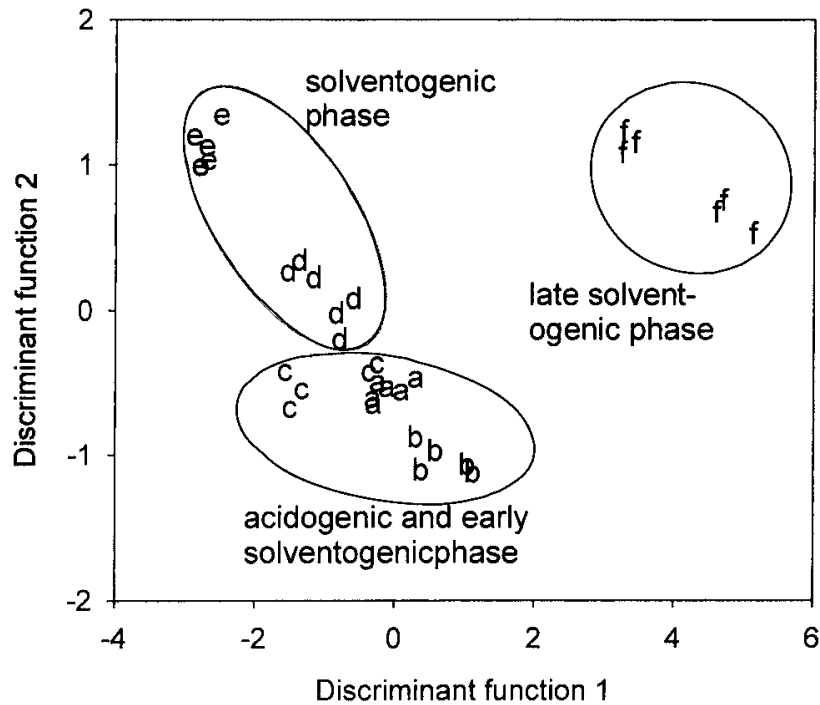

Figure 6 Discriminant function analysis of the spectra over the growth and production curve of $C$. beijerinckii NCIMB 8052. Sampling time points are marked with letters "a" to "f" corresponding to those shown in Figure 4. Each data point refers to a spectrum. Two biological replicates (fermentation experiments) were sampled. Three replicate spectra were taken from each sample.

\section{Mixing experiment}

To determine whether spectroscopic methods could be employed to determine the proportion of degenerated bacteria in a liquid culture medium, artificial mixtures of cells from cultures of $C$. beijerinckii NCIMB 8052, the wild-type strain and strain DN, the degenerate variant were analysed. They were grown separately in liquid medium, inoculated from liquid precultures derived from single colonies. From these cultures, cell suspensions were sampled at two different points in the growth and production curve and mixed in different known ratios. The resulting mixtures were harvested and treated as above to obtain FT-IR spectra.

The culture conditions at which the cells were harvested before mixing are given in Table 1. Sample 1 was taken at the end of the acidogenic phase of the normal culture, just after solvent production had started. The second sample was taken when the culture of the normal strain had produced a substantial amount of solvents (and that of the degenerate variant had produced a substantial amount of acids). Cell densities in the different cultures were about the same in sample 1. In sample 2, the optical density of the normal culture was about twice that of the degenerate culture. Some of the spectra

Table 1 Culture variables for the cell samples used in the mixing experiment

\begin{tabular}{|c|c|c|c|c|}
\hline Time $(\mathrm{h})$ & $\mathrm{OD}_{615 \mathrm{~nm}}$ & $\mathrm{pH}$ & Butanol (g/1) & Total acids $(\mathrm{g} / \mathrm{l})$ \\
\hline \multicolumn{5}{|c|}{ Normal strain C. beijerinckii NCIMB 8052} \\
\hline 21 & 2.3 & 5.15 & 1.0 & 2.8 \\
\hline 43 & 6.1 & 5.82 & 11.2 & 1.7 \\
\hline 71 & 9.0 & 5.79 & 10.6 & 2.3 \\
\hline \multicolumn{5}{|c|}{ Degenerate variant $D N$} \\
\hline 21 & 1.9 & 4.7 & $<0.1$ & 3.7 \\
\hline 43 & 2.7 & 4.5 & 0.4 & 6.6 \\
\hline
\end{tabular}

from the artificial mixtures of time 1 (starting at $0 \%$ degenerate cells, and incremental steps of $10 \%$ ) and the known ratios were used as training set (input) for a mathematical model formed using the partial least squares (PLS) algorithm. Another set of spectra (starting at 5\% degenerate cells, and incrementing in steps of 10\%) was used for cross-validation. A third set, the spectra from time 2, were used as independent test set. The results of this quantification are given in Figure 7.

As a measure of accuracy for the predictions, the root mean square error was calculated for the data sets. Predictions inside the training set had a root mean square error of $5.7 \%$, those of the test set of $9.1 \%$. Predictions of the ratio of degenerate cells in the culture were possible from sample 1 across to sample 2, on which the model had not been trained, with somewhat lower accuracy (root mean square error of $12.4 \%$ ), however. The model therefore appears to detect differences in spectra, which are relevant to degeneration, even in the presence of potentially interfering changes over the bacterial growth curve. The accuracy is probably sufficient to detect the appearance of a degenerate subpopulation, e.g., during continuous cultivation at a comparatively early stage.

Figure 8 shows representative spectra of the normal and degenerate strain, from the first sampling time. The observed differences are in the structure and quantity of the amide II band around $1545 \mathrm{~cm}^{-1}$, and especially in the carbohydrate region (1200-900 $\left.\mathrm{cm}^{-1}\right)$. The wild type has a much higher peak at $1156 \mathrm{~cm}^{-1}$ and a different fine structure between 1033 and $997 \mathrm{~cm}^{-1}$. This may be related to granulose formation in the wild type, but also to differences in cell wall structure. Comparison of the spectra in Figures 5 and 8 indicates why the quantification model can differentiate between the changes by degeneration and by the normal cell cycle - the spectral features involved are different.

Samples of the artificial mixtures of normal and degenerate cells were plated on CBM medium. The results were in line with the

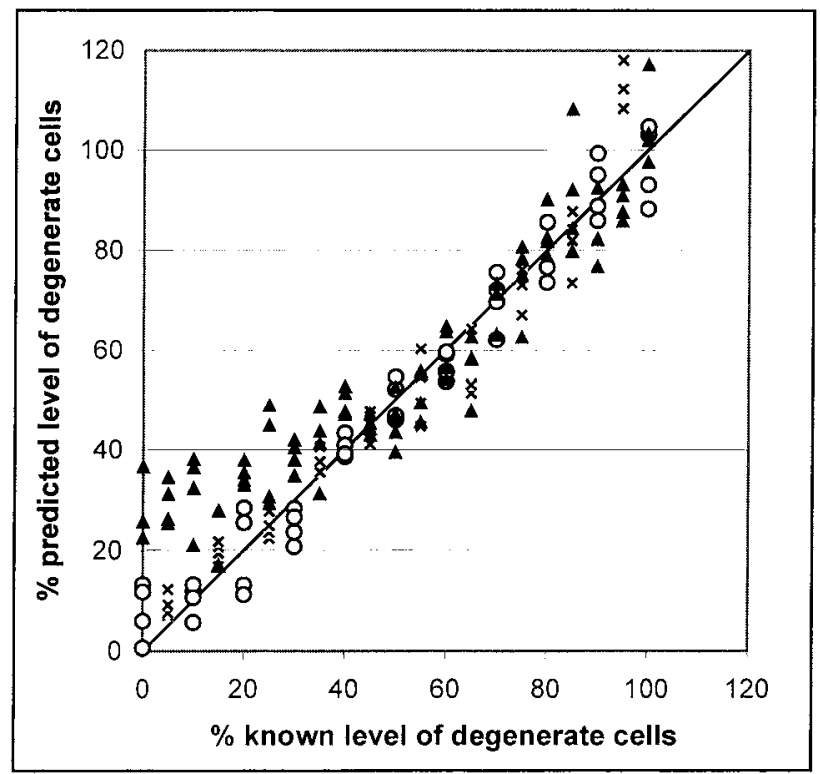

Figure 7 Results of the quantification by partial least squares of normal and degenerate cells in an artificially mixed bacterial population. The whole recorded wave number range was used. Quantification by partial least squares on time 1 (four factors). $O$, training set (prediction of time 1 ). $\times$, test set (prediction of time 1 , unknown samples). $\boldsymbol{\Delta}$, independent test set (prediction of time 2). 


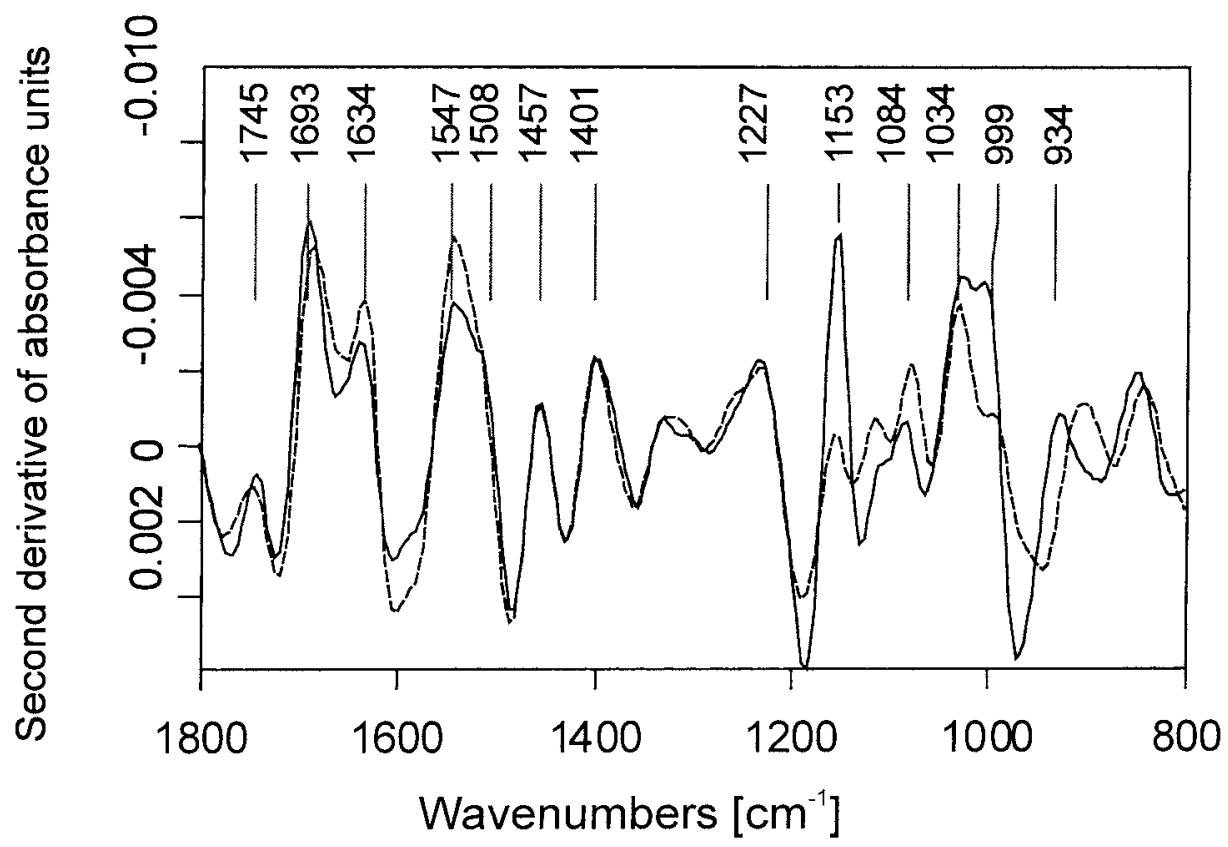

Figure 8 Second - derivative spectra of biomass from cultures of the wild type of NCIMB 8052 and strain DN, the degenerate variant. Solid line, wild type $(0 \%$ degenerate $)$; dashed line, strain variant DN (100\% degenerate cells $)$. Spectra from time $1(21 \mathrm{~h})$ are shown.

known mixing ratios (data not shown). There are two distinct advantages of the FT-IR spectroscopic method as compared with the plate count technique. Firstly, analysis by the FT-IR method takes less than $1 \mathrm{~h}$ from sampling, whereas colony formation in the plate count assay takes at least 2 days. The FT-IR method is fast enough to be usefully applied in process control. Secondly, the FTIR method analyses all the cells present in the culture, whereas the plate count assay detects only culturable cells. It is well known that in the later phases of the acetone-butanol-ethanol (ABE) fermentation, the majority of cells, although metabolically active in solvent production, no longer form colonies, except through the sporulation cycle. In these experiments, despite the use of the anaerobic cabinet for all steps, the recovery of cells to form colonies on plates (i.e., plating efficiency) was $1 \%$ or less, which casts serious doubt as to whether the results obtained are representative of the entire bacterial population.

The mixing experiments shown here are a model for the analysis of mixed cultures. Certainly the final proof of the method would be to monitor a continuous cultivation where a degeneration occurs spontaneously. The results of the spectroscopic quantification can be compared with plate counts; however, it is necessary to keep in mind the problems with this technique as discussed above.

\section{Conclusions and outlook}

The formation of degenerate variants in solventogenic clostridia can be detected by FT-IR spectroscopy of bacterial cells. Colonies of degenerate variants on solid medium, as well as samples from variants grown in liquid medium, show spectra that are distinct from those of the parental strain. The proportion of degenerate cells in a liquid culture was quantified by evaluation of FT-IR spectra from artificial mixtures. The spectra of $C$. beijerinckii NCIMB 8052 cells changed remarkably according to their physiological status. In the course of a normal batch culture, the spectral changes readily allow distinction of the acidogenic and solventogenic phases. These changes due to the growth cycle in batch culture can be distinguished from those that occur in degenerate variants.

This opens a new perspective for process monitoring and control in the continuous acetone-butanol fermentation process via nearreal time monitoring of population dynamics. Moreover, basic studies on mechanisms of Clostridium strain degeneration can be supported by this fast method to detect population changes, enabling dynamic studies on the degenerative process.

\section{Acknowledgements}

We thank the EBS committee of the UK BBSRC and the European Commission (FAIR-CT96-1201) for financial support.

\section{References}

1 Adler HI and W Crow. 1987. A technique for predicting the solventproducing ability of Clostridium acetobutylicum. Appl Environ Microbiol 53: 2496-2499.

2 Bahl H, W Andersch, K Brown and G Gottschalk. 1982. Effects of pH and butyrate concentration on the production of acetone and butanol by Clostridium acetobutylicum grown in continuous culture. Eur J Appl Microbiol Biotechnol 14: 17-20.

3 Barbeau JY, R Marchal and JP Vandecasteele. 1988. Conditions promoting stability of solventogenesis or culture degeneration in continuous fermentations of Clostridium acetobutylicum. Appl Microbiol Biotechnol 29: 447-455.

4 Bryant DL and HP Blaschek. 1988. Buffering as a means for increasing growth and butanol production by Clostridium acetobutylicum. $J$ Ind Microbiol 3: 49-55.

5 Clarke KG, GS Hansford and DT Jones. 1988. Nature and significance of oscillatory behavior during solvent production by Clostridium acetobutylicum in continuous culture. Biotechnol Bioeng 32: 538-544.

6 Cornillot E and P Soucaille. 1996. Solvent-forming genes in clostridia. Nature 380: 489. 
7 Cornillot E, RV Nair, ET Papoutsakis and P Soucaille. 1997. The genes for butanol and acetone formation in Clostridium acetobutylicum ATCC 824 reside on a large plasmid whose loss leads to degeneration of the strain. J Bacteriol 179: 5442-5447.

8 Dürre P. 1998. New insights and novel developments in clostridial acetone/butanol/isopropanol fermentation. Appl Microbiol Biotechnol 49: $639-648$.

9 Ennis B and IS Maddox. 1989. Production of solvents (ABE fermentation) from whey permeat by continuous fermentation in a membrane bioreactor. Bioprocess Eng 4: 27-34.

10 Evans VJ, H Liyanage, A Ravagnani, M Young and ER Kashket. 1998. Truncation of peptide deformylase reduces the growth rate and stabilizes solvent production in Clostridium beijerinckii NCIMB 8052. Appl Environ Microbiol 64: 1780-1785.

11 Finn RK and JE Nowrey. 1958. A note on the stability of clostridia when held in continuous culture. Appl Microbiol 7: 29-32.

12 Gapes JR. 2000. The economics of the acetone-butanol fermentation - theoretical and market considerations. J Mol Microbiol Biotechnol 2: $27-32$.

13 Gapes JR, D Nimcevic and A Friedl. 1996. Long-term continuous cultivation of Clostridium beijerinckii in a two-stage chemostat with on-line solvent removal. Appl Environ Microbiol 62: 3210-3219.

14 Goodacre R, ÉM Timmins, P Rooney, J Rowland and DB Kell. 1996. Rapid identification of Streptococcus and Enterococcus species using diffuse reflectance-absorbance Fourier transform infrared spectroscopy and artificial neural networks. FEMS Microbiol Lett 140: $233-239$.

15 Griffiths PR and JA de Haseth. 1986. Fourier transform infrared spectrometry. New York: Wiley.

16 Hayashida S and S Yoshino. 1990. Degeneration of solventogenic Clostridium caused by a defect in NADH generation. Agric Biol Chem 54: $427-435$.

17 Jolliffe IT. 1986. Principal Component Analysis. New York: SpringerVerlag.

18 Jones A, AD Shaw, GJ Salter, G Bianchi and DB Kell. 1998. The exploitation of chemometric methods in the analysis of spectroscopic data: application to olive oils. In: Hamilton RJ (Ed.), Lipid Analysis of Oils and Fats. Chapman \& Hall, London, pp. 317-376.

19 Jones DT and DR Woods. 1986. Acetone-butanol fermentation revisited. Microbiol Rev 50: 484-524.

20 Kashket ER and ZY Cao. 1993. Isolation of a degeneration-resistant mutant of Clostridium acetobutylicum NCIMB 8052. Appl Environ Microbiol 59: 4198-4202.

21 Kashket ER and ZY Cao. 1995. Clostridial strain degeneration. FEMS Microbiol Rev 17: 307-315.

22 Kutzenok A and M Aschner. 1952. Degenerative processes in a strain of Clostridium butylicum. J Bacteriol 64: 829-836.

23 Liyanage H, P Holcroft, VJ Evans, S Keis, SR Wilkinson, ER Kashket and M Young. 2000. A new insertion sequence, ISCb1, from Clostridium beijerinckii NCIMB 8052. J Mol Microbiol Biotechnol 2: $107-113$.

24 Maddox IS, E Steiner, S Hirsch, S Wessner, NA Gutierrez, JR Gapes and KC Schuster. 2000. The cause of acid crash and acidogenic fermentation process. J Mol Microbiol Biotechnol 2: 95-100.

25 Manly BFJ. 1994. Multivariate Statistical Methods: A Primer. Chapman \& Hall, London.

26 Martens $\mathrm{H}$ and $\mathrm{T}$ Næs. 1989. Multivariate Calibration. Wiley, Chichester.

27 Naumann D. 2000. FT-Infrared and FT-Raman spectroscopy in biomedical research. In: Gremlich H-U, Yan B (Eds.), Infrared and Raman spectroscopy of biological materials. Marcel Dekker, New York, pp. 323-378.

28 Naumann D, V Fialja, H Labischinski and P Giesbrecht. 1988. The rapid differentiation and identification of pathogenic bacteria using Fourier transform-infrared spectroscopic and multivariate statistical analysis. J Mol Struct 174: 165-170.

29 O'Brien RW and JG Morris. 1971. Oxygen and the growth and metabolism of Clostridium acetobutylicum. J Gen Microbiol 68 : 307-318.

30 Reardon KF and JE Bailey. 1989. Effects of $\mathrm{pH}$ and added metabolites on bioconversions by immobilized non-growing Clostridium acetobutylicum. Biotechnol Bioeng 34: 825-837.

31 Rogers P, G Gottschalk and D Jones. 1993. Biochemistry and regulation of acid and solvent production in clostridia. In: Woods D (Ed.), The Clostridia and Biotechnology. Butterworth-Heinemann, Stoneham, MA, pp. 25-50.

32 Savitzky A and MJE Golay. 1964. Smoothing and differentiation of data by simplified least squares procedures. Anal Chem 36: $1627-1633$.

33 Schuster KC. 2000. Introduction: Fermentation Symposium. J Mol Microbiol Biotechnol 2: 3-4.

34 Schuster KC, F Mertens and JR Gapes. 1999. FTIR spectroscopy applied to bacterial cells as a novel method for monitoring complex biotechnological processes. Vib Spectrosc 19: 467-477.

35 Schuster KC, R van den Heuvel, NA Gutierrez and IS Maddox. 1998. Development of markers for product formation and cell cycle in batch cultivation of Clostridium acetobutylicum ATCC 824. Appl Microbiol Biotechnol 49: 669-676.

36 Seasholtz MB and BR Kowalski. 1992. The effect of mean centering on prediction in multivariate calibration. J Chemometrics 6: 103-111.

37 Stim-Herndon KP, R Nair, ET Papoutsakis and GN Bennett. 1996 Analysis of degenerate variants of Clostridium acetobutylicum ATCC 824. Anaerobe 2: $11-18$.

38 Timmins ÉM, SA Howell, BK Alsberg, WC Noble and R Goodacre 1998. Rapid differentiation of closely related Candida species and strains by pyrolysis mass spectrometry and Fourier transform infrared spectroscopy. J Clin Microbiol 36: 367-374.

39 Wold H. 1966. Estimation of principal components and related models by iterative least squares. In: Krisnaiah KR (Ed.), Multivariate Analysis. Academic Press, New York, pp. 391-420.

40 Woolley RC and JG Morris. 1990. Stability of solvent production by Clostridium acetobutylicum in continuous culture: strain differences. J Appl Bacteriol 69: 718-728. 